Research Paper

\title{
The postoperative lean body mass loss at one month leads to a poor survival in patients with locally advanced gastric cancer
}

\author{
Toru Aoyama ${ }^{1,2}{ }^{\varpi}$, Takaki Yoshikawa ${ }^{1,2}$, Yukio Maezawa ${ }^{1,2}$, Kazuki Kano, ${ }^{1,2}$, Masakatsu Numata1,2, Kentaro \\ Hara $^{1,2}$, Keisuke Komori ${ }^{1,2}$, Takanobu Yamada ${ }^{1,2}$, Tsutomu Hayashi 1,2, Tsutomu Sato ${ }^{1,2}$, Hiroshi \\ Tamagawa ${ }^{1,2}$, Norio Yukawa ${ }^{2}$, Yasushi Rino ${ }^{2}$, Munetaka Masuda ${ }^{2}$, Takashi Ogata1 ${ }^{1}$ Haruhiko Cho1, \\ Takashi Oshima ${ }^{1,2}$ \\ 1. Department of Gastrointestinal Surgery, Kanagawa Cancer Center, Yokohama, Japan \\ 2. Department of Surgery, Yokohama City University, Yokohama, Japan \\ $\triangle$ Corresponding author: Toru Aoyama, Department of Surgery, Yokohama City University, 3-9 Fukuura Kanazawa-ku, Ykokohama 236-0004, Japan. \\ (c) Ivyspring International Publisher. This is an open access article distributed under the terms of the Creative Commons Attribution (CC BY-NC) license \\ (https://creativecommons.org/licenses/by-nc/4.0/). See http://ivyspring.com/terms for full terms and conditions.
}

Received: 2018.10.15; Accepted: 2019.04.13; Published: 2019.05.28

\begin{abstract}
Aims: We previously demonstrated that a loss of lean body mass loss at one month after gastrectomy was an independent risk factor for the continuation of adjuvant chemotherapy with S- 1 . However, it is unclear whether or not lean body mass loss after gastrectomy leads to a poor survival through poor compliance to adjuvant chemotherapy with S-1.

Methods: The recurrence free survival (RFS) overall survival (OS) and were examined in 115 patients who underwent curative gastrectomy and were pathologically diagnosed with stage II or III gastric cancer and who received postoperative adjuvant chemotherapy with S-1 between May 2011 and September 2016.

Results: The median follow-up period was 40.6 months. The RFS rates at 5 years after surgery were $57.8 \%$ in the lean body mass loss $\geq 5 \%$ group and $73.5 \%$ in the lean body mass loss $<5 \%$ group. The univariate and multivariate analyses for the disease free survival (RFS) demonstrated that a lean body mass loss $>5 \%$ was a significant risk factor. The OS rates at 5 years after surgery were $72.0 \%$ in the lean body mass loss $\geq 5 \%$ group and $77.3 \%$ in the lean body mass loss $<5 \%$ group. The OS was slightly worse in the lean body mass loss $\geq 5 \%$ group than in the lean body mass loss $<5 \%$ group $(p=0.2062)$.

Conclusions: The lean body mass loss at one month, which is closely associated with poor S- 1 compliance, was an important risk factor for the RFS. A prospective cohort study is necessary to confirm whether or not the lean body mass loss affects the gastric cancer survival.
\end{abstract}

Key words: gastric cancer - adjuvant chemotherapy- lean body mass loss -recurrence free survival

\section{Introduction}

Gastric cancer accounted for 951,600 new cancer cases and 723,100 deaths in $2012^{1}$. Complete resection is essential to cure locally advanced gastric cancer. At present, the standard treatments for locally advanced gastric cancer in Asia, Europe and the United States are D2 gastrectomy followed by adjuvant chemotherapy, surgery with pre- and postoperative chemotherapy, and surgery with postoperative chemoradiotherapy, respectively ${ }^{2-8}$. Thus, adjuvant treatment plays a crucial role in improving the gastric cancer survival.

We recently reported that the loss of lean body mass at one month after gastrectomy was an independent risk factor for the continuation of adjuvant chemotherapy with S-1 ${ }^{9}$. The 6-month continuation rate of S-1 treatment was $91.7 \%$ in the 
patients with a loss of lean body mass $<5 \%$ and $66.3 \%$ in patients with a loss of lean body mass $\geq 5 \%$ $(\mathrm{P}=0.031)$. Poor compliance with adjuvant chemotherapy might lead to a poor survival ${ }^{10}$. Furthermore, marginally significant differences were noted in the incidence of grade 3 toxicities between the patients with a loss of lean body mass $<5 \%$ and a loss of lean body mass $\geq 5 \%$ ( $18.9 \%$ vs. $42.9 \%$, $\mathrm{P}=0.050)$. Although the loss of lean body mass at one month after gastrectomy can affect the continuation of adjuvant chemotherapy, the survival impact of this loss is unclear. A previous study showed that the efficacy of adjuvant chemotherapy is weakened when the treatment is insufficient ${ }^{11}$.

Given these previous findings, we hypothesized that the loss of lean body mass at one month after gastrectomy would not only decrease the rate of continuation of S-1 adjuvant chemotherapy but also have some impact on the gastric cancer survival. To confirm our hypothesis, we evaluated whether or not the loss of lean body mass at one month after gastrectomy is a prognostic factor for patients with locally advanced gastric cancer who underwent curative gastrectomy followed by adjuvant chemotherapy with S-1.

\section{Patients and Methods}

\section{Patients}

The patients were selected from the prospective database of the Kanagawa Cancer Center, Department of Gastrointestinal Surgery, Yokohama, Japan, according to the following criteria; histologically-proven gastric adenocarcinoma, (2) curative lymph node dissection for gastric cancer as a primary treatment between May 2011 and September 2016, (3) stage II or III disease diagnosed pathologically according to the $14^{\text {th }}$ edition of the general rules for gastric cancer published by the Japanese Gastric Cancer Association 12, (4) started adjuvant chemotherapy of S-1 at a dose of $80 \mathrm{mg} / \mathrm{m}^{2}$, and (5) underwent a body composition analysis within 1 week before surgery and at 1 month after surgery.

\section{Surgical procedures and perioperative care}

All patients received distal or total gastrectomy with nodal dissection for gastric cancer ${ }^{13}$. In principle, D1 or a D1+ lymphadenectomy is indicated for cT1N0 tumors, and D2 is applied for $\mathrm{cN}+$ or cT2-T4 tumors, regardless of the approach. Spleen-preserving D2 total gastrectomy was permitted in this study.

\section{Postoperative care}

As described in our previous report, the patients received perioperative care according to the ERAS protocol ${ }^{14}$. In brief, the patients were allowed to eat until midnight on the day before surgery and were required to drink a rehydration solution by three hours before surgery. The nasogastric tube was removed immediately after surgery. The oral intake was initiated on postoperative day (POD) 1, beginning with water and an oral nutritional supplement. The patients began to eat solid food on POD 2, starting with rice gruel and soft food on POD 3 and advancing in three steps to regular food intake on POD 7. The patients were discharged when they had achieved adequate pain relief and soft food intake and exhibited normal laboratory data on POD 7. We did not use any postoperative medications to increase appetite, bowel movement or nutrition supplement for patients in this study. Furthermore, in the present study, although all of the patients received the same aftercare, including a physical examination and laboratory tests, after surgery at the outpatient clinic, the patients did not receive any oral nutrition supplements to help them avoid lean mass loss.

\section{Adjuvant treatment with S-1}

The patients received S-1 chemotherapy at 80-120 mg/body per day according to the body surface area (BSA): BSA $<1.25 \mathrm{~m}^{2}, 80 \mathrm{mg} /$ day; $1.25<$ $\mathrm{BSA}<1.5 \mathrm{~m}^{2}, 100 \mathrm{mg} /$ day; $1.5 \mathrm{~m}^{2}<\mathrm{BSA}, 120 \mathrm{mg} /$ day 4 . The planned period of S-1 treatment was one year, except for the patients who were registered to in the optimal period of adjuvant S-1 chemotherapy for pathological stage II gastric cancer patients who underwent D2 gastrectomy trial, OPAS-1 (Optimal period of adjuvant S-1 chemotherapy, a multi-center phase III trial for pathological stage II gastric cancer patients who underwent D2 gastrectomy to confirm non-inferiority of the recurrence-free survival of the test arm of 4 courses of S-1 adjuvant chemotherapy against the control arm of 8 courses of S-1) after February $2012{ }^{15}$. The doses were modified as follows: when adverse reactions appeared, the dose was reduced from 120 to $100 \mathrm{mg} /$ day or from 100 to 80 $\mathrm{mg} /$ day or the administration was temporarily discontinued. Treatment was discontinued when the patient showed recurrence of disease or adverse reactions that were uncontrollable even by dose modification or the temporary withdrawal of drug administration.

\section{Follow-up}

The patients were followed up at an outpatient clinic. They received no treatments other than adjuvant chemotherapy with S-1 until recurrence. Hematological tests and physical examinations were performed at least every two to three weeks during S-1 treatment and at least every six months for five 
years after the completion of S-1 treatment. The CEA and CA19-9 tumor marker levels were checked at least every six months for five years. Patients underwent computed tomography every six months during the first three years after surgery and then every year until five years after surgery.

\section{The body composition analysis}

The segmental body composition was analyzed using the Tanita MC-190EM bioelectrical impedance analyzer (Tanita, Tokyo, Japan), which provides relative information regarding the amount of lean and fat tissue in the trunk area and each limb as well as the overall body composition and hydration status. The body weight and composition were evaluated by a bioelectrical impedance analyzer within one week before surgery and at one month after surgery. In addition, time for body composition analyses was morning before breakfast because body water is influenced by oral intake.

\section{Evaluations and statistical analyses}

The lean body mass loss was defined as follows: $\%$ loss of lean body mass $=$ (preoperative lean body mass - lean body mass at 1 month after surgery) $\times 100$ / preoperative lean body mass. The preoperative lean body mass was measured within one week before the surgery.

The overall survival (OS) was defined as the period between surgery and death. The recurrence-free survival (RFS) was defined as the period between surgery and recurrence or death, whichever came first. Survival curves were calculated using the Kaplan-Meier method and compared by the log-rank test. Cox's proportional hazards model was used to perform the univariate and multivariate survival analyses. $P$ values of $<0.05$ were considered to indicate statistical significance. The SPSS software program (v11.0J Win; SPSS, Chicago, IL, USA) was used to perform all of the statistical analyses. This study was approved by the institutional review board of Kanagawa Cancer Center.

\section{Results}

\section{Patients' background characteristics}

A total of 888 patients underwent surgical resection and received a perioperative body composition analysis between May 2011 and September 2016. A flow diagram of the 888 patients is shown in Figure 1. One-hundred and fifteen of these patients were eligible for the present study. There were more patients who had lympho-vascular invasion in the lean body mass loss $\geq 5 \%$ group than in the lean body mass loss $<5 \%$ group $(87.3 \%$ vs. $71.2 \%$, $\mathrm{p}=0.031$ ). Furthermore, the incidence of pathologically diffuse type was higher in the lean body mass loss $\geq 5 \%$ group than in the lean body mass loss $<5 \%$ group $(52.4 \%$ vs. $26.9 \%, p=0.006)$ (Table 1$)$. However, there were no marked differences in the depth of tumor invasion or rate of lymph node metastasis between the two groups.

\section{Survival analyses}

The median follow-up period was 40.6 months (range: 13.6-80.4 months). The RFS rates at 3 and 5 years after surgery were $60.3 \%$ and $57.8 \%$ in the lean body mass loss $\geq 5 \%$ group and $80.9 \%$ and $73.5 \%$ in the lean body mass loss $<5 \%$ group, respectively. The recurrence free survival (RFS) was therefore significantly worse in the lean body mass loss $\geq 5 \%$

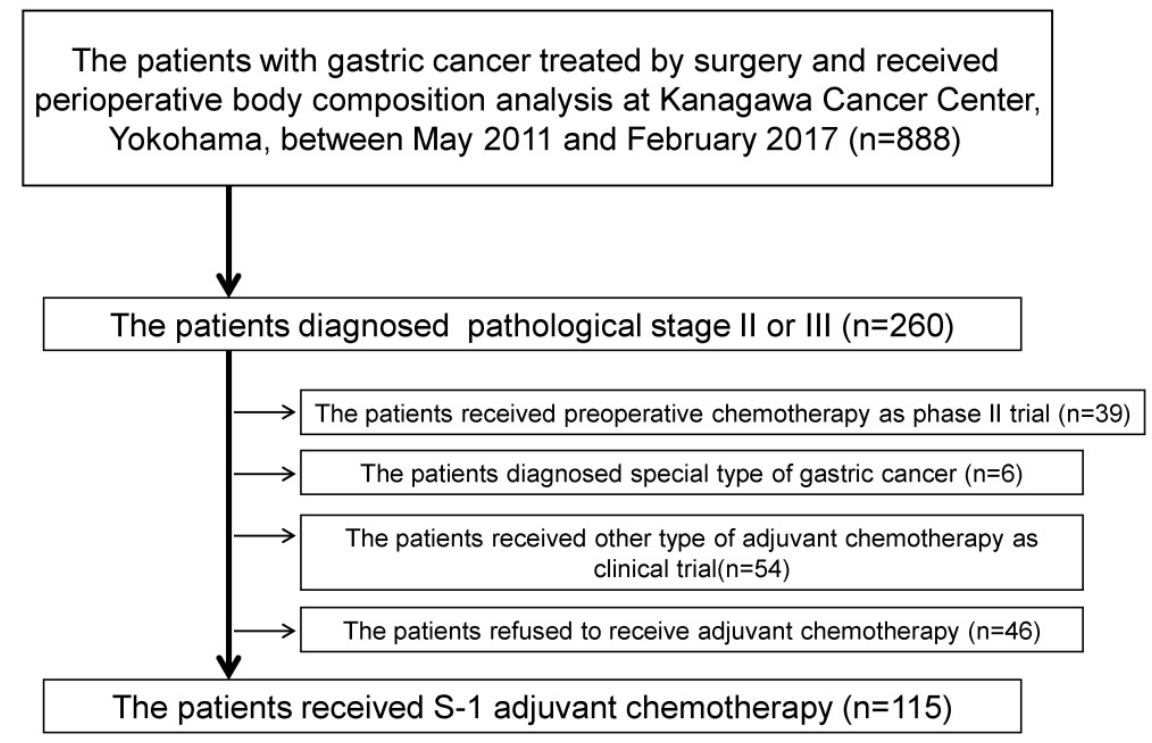

Figure 1. Flow diagram of the present study 
group than in the lean body mass loss $<5 \%$ group $(p=0.045)$. The RFS curves are shown in Figure 2. The univariate and multivariate analyses for the RFS demonstrated that lean body mass loss $\geq 5 \%$ was a significant risk factor. In contrast, $\mathrm{T}$ factor, $\mathrm{N}$ factor, and postoperative surgical complications were not selected as significant prognosticators for the RFS in either the univariate or multivariate analysis. The hazard ratio for the RFS was 2.076 (95\% confidence interval, 1.016 to 4.239; $\mathrm{P}$ value $=0.045$, Table 2) .

The OS rates at 3 and 5 years after surgery were $74.8 \%$ and $72.0 \%$ in the lean body mass loss $\geq 5 \%$ group and $85.4 \%$ and $77.3 \%$ in the lean body mass loss $<5 \%$ group, respectively. The OS was slightly worse in the lean body mass loss $\geq 5 \%$ group than in the lean body mass loss $<5 \%$ group ( $p=0.2062$ ). The OS curves are shown in Figure 3. The univariate analyses for the OS demonstrated that the lean body mass loss $\geq 5 \%$ was not a significant risk factor for the OS.

When comparing the sites of first relapse, the incidence of hematological recurrence was marginally significantly higher in the lean body mass loss $\geq 5 \%$ group than in the lean body mass loss $<5 \%$ group (Table 3).
Table 1. Patients characteristics

\begin{tabular}{|c|c|c|c|c|}
\hline & All cases & $\begin{array}{l}\text { Patients with } 5 \% \text { or } \\
\text { more LBM loss at } \\
\text { 1month }\end{array}$ & $\begin{array}{l}\text { Patients without } 5 \% \text { or } \\
\text { more LBM loss at } 1 \text { month }\end{array}$ & $\begin{array}{l}P \\
\text { value }\end{array}$ \\
\hline & $\begin{array}{l}\mathrm{N}=115 \\
(\%)\end{array}$ & $\mathrm{N}=52(\%)$ & $\mathrm{N}=63(\%)$ & \\
\hline $\begin{array}{l}\text { Age (year) } \\
\text { (range) }\end{array}$ & $\begin{array}{l}70 \\
(36-85)\end{array}$ & $\begin{array}{l}68.5 \\
(36-85)\end{array}$ & $\begin{array}{l}71 \\
(45-85)\end{array}$ & 0.140 \\
\hline Gender & & & & 0.611 \\
\hline Male & $78(67.8)$ & $34(65.4)$ & $44(69.8)$ & \\
\hline Female & $37(32.2)$ & $18(34.6)$ & $19(30.2)$ & \\
\hline Tumor loc & cation & & & 0.097 \\
\hline $\begin{array}{l}\text { Upper } \\
\text { third }\end{array}$ & $35(30.4)$ & $11(21.2)$ & $24(38.1)$ & \\
\hline $\begin{array}{l}\text { Middle } \\
\text { third }\end{array}$ & $39(33.9)$ & $18(34.6)$ & $21(33.3)$ & \\
\hline $\begin{array}{l}\text { Lower } \\
\text { third }\end{array}$ & $41(35.7)$ & $23(44.2)$ & $18(28.6)$ & \\
\hline \multicolumn{4}{|c|}{ Type of surgery } & 0.674 \\
\hline DG & $65(56.5)$ & $33(63.5)$ & $32(50.8)$ & \\
\hline TG & $40(43.5)$ & $19(36.5)$ & $31(49.2)$ & \\
\hline \multicolumn{4}{|c|}{ Pathological $\mathrm{T}$ factor } & 0.437 \\
\hline$-\mathrm{T} 3$ & $51(44.3)$ & $21(40.4)$ & $30(47.6)$ & \\
\hline T4- & $64(55.7)$ & $31(59.6)$ & $33(52.4)$ & \\
\hline \multicolumn{4}{|c|}{ Pathological $\mathrm{N}$ factor } & 0.147 \\
\hline Negative & $24(20.9)$ & $14(26.9)$ & $10(15.9)$ & \\
\hline Positive & $91(79.1)$ & 38 (73.1) & $53(84.1)$ & \\
\hline \multicolumn{4}{|c|}{ Pathological type } & 0.006 \\
\hline Intestinal & $68(59.1)$ & 38 (73.1) & $30(47.6)$ & \\
\hline Diffuse & $47(40.9)$ & $14(26.9)$ & $33(52.4)$ & \\
\hline \multicolumn{4}{|c|}{$\begin{array}{l}\text { Lymph-vascular } \\
\text { invasion }\end{array}$} & 0.031 \\
\hline Negative & $23(20.0)$ & $15(28.8)$ & $8(12.7)$ & \\
\hline Positive & $92(80.0)$ & $37(71.2)$ & $55(87.3)$ & \\
\hline \multicolumn{4}{|c|}{$\begin{array}{l}\text { Postoperative } \\
\text { complication }\end{array}$} & 0.245 \\
\hline No & $87(75.7)$ & $42(80.8)$ & $45(71.4)$ & \\
\hline Yes & $28(24.3)$ & $10(19.2)$ & $18(28.6)$ & \\
\hline
\end{tabular}

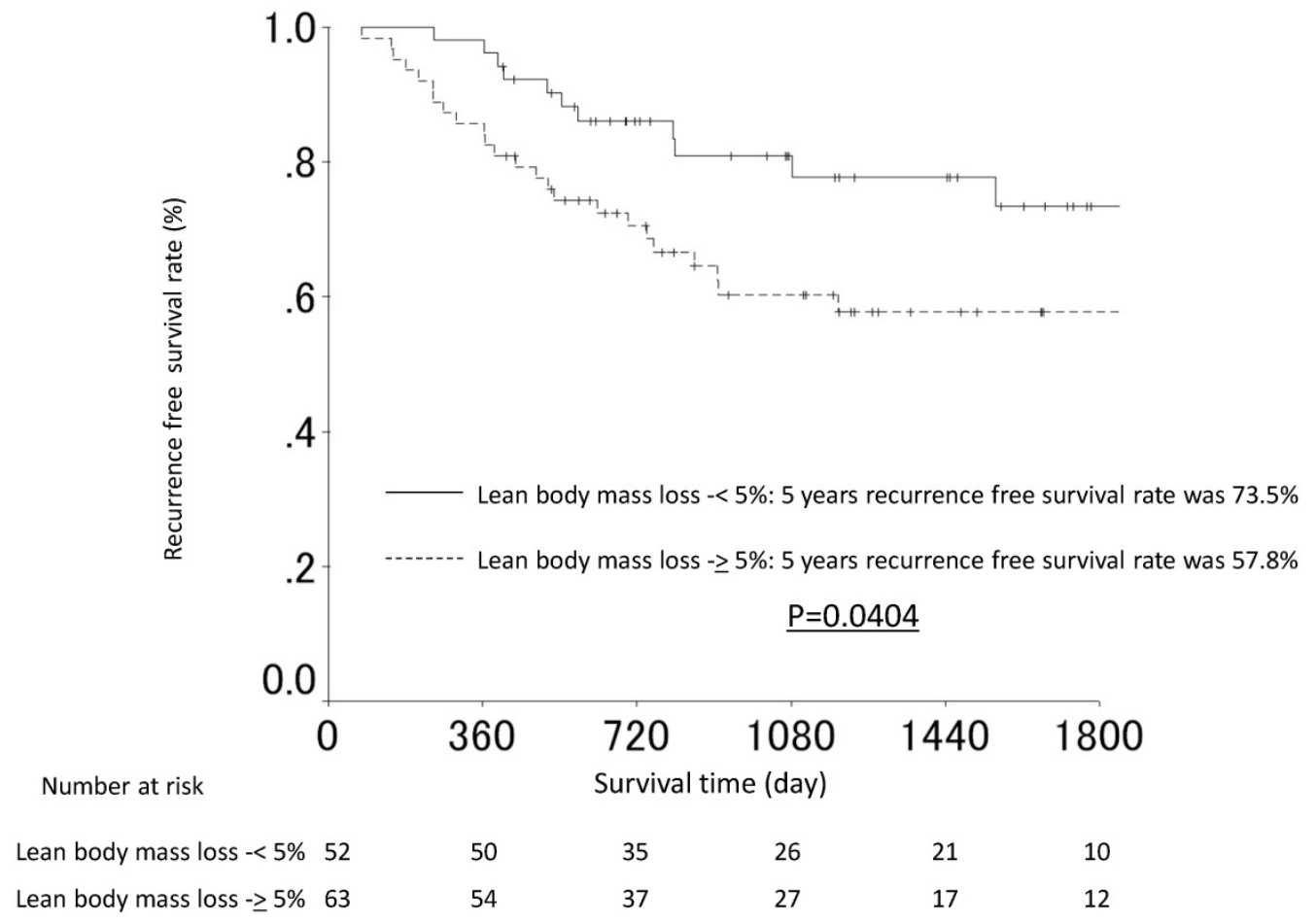

Figure 2. The recurrence-free survival curves of the patients in the lean body mass loss $\geq 5 \%$ and the lean body mass loss $<5 \%$ groups. 


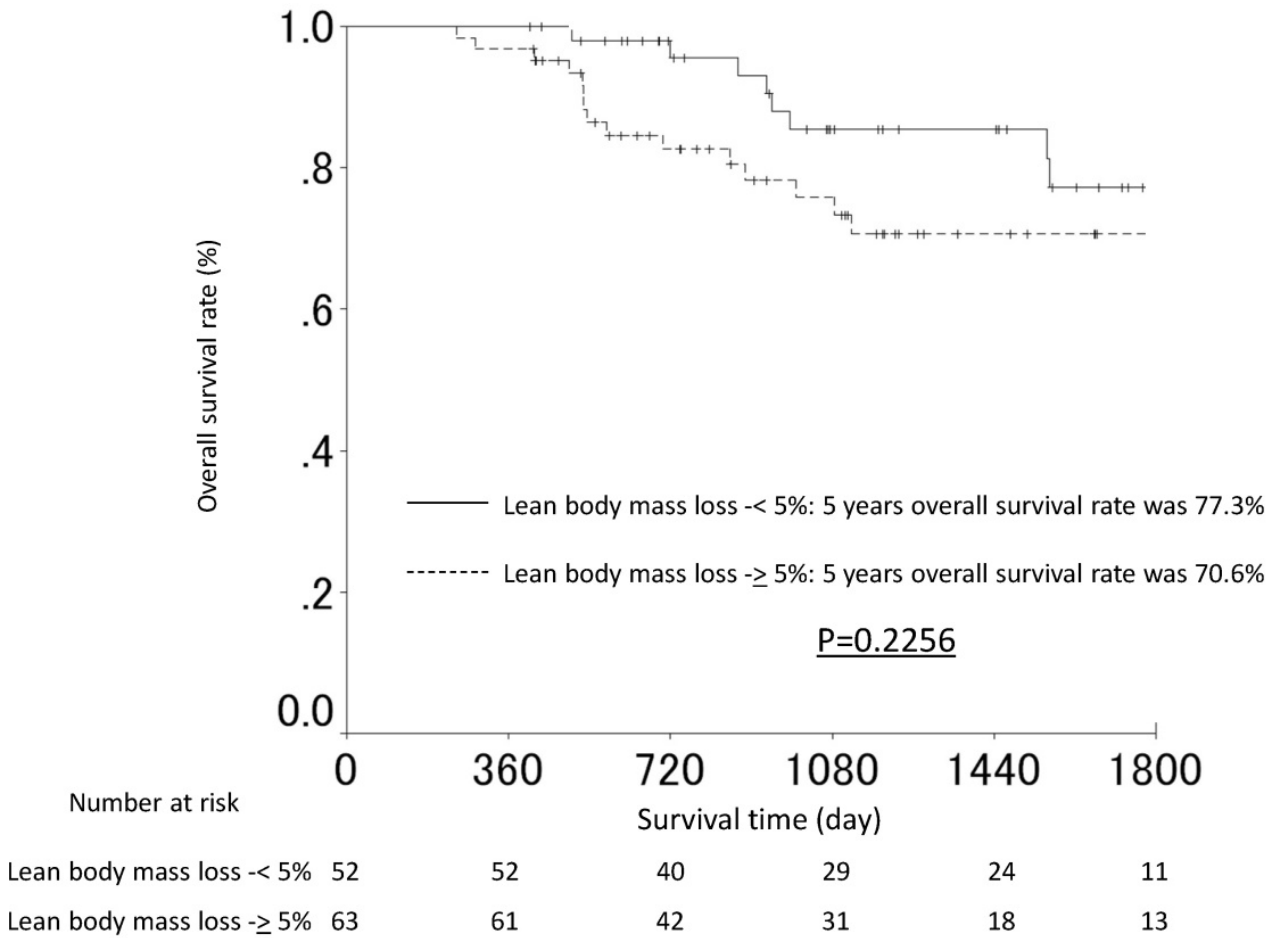

Figure 3. The overall survival curves of the patients in the lean body mass loss $\geq 5 \%$ and the lean body mass loss $<5 \%$ groups.

Table 2. The univariate and multivariate Cox proportional hazards analysis of clinicopathological factors for recurrence free survival.

\begin{tabular}{|c|c|c|c|c|c|c|c|}
\hline \multirow{2}{*}{ Factor } & \multicolumn{3}{|c|}{ Univariate } & \multirow[b]{2}{*}{$P$ value } & \multicolumn{2}{|c|}{ Multivariate } & \multirow[b]{2}{*}{$\begin{array}{l}\mathrm{P} \\
\text { value }\end{array}$} \\
\hline & Number & HR & $95 \% \mathrm{CI}$ & & HR & $95 \% \mathrm{CI}$ & \\
\hline \multicolumn{2}{|l|}{ Age (years) } & & & 0.729 & & & \\
\hline$<70$ years & 53 & 1.000 & & & & & \\
\hline$\geq 70$ years & 62 & 1.126 & $0.576-2.199$ & & & & \\
\hline \multicolumn{2}{|l|}{ Gender } & & & 0.624 & & & \\
\hline Female & 37 & 1.000 & & & & & \\
\hline Male & 78 & 1.196 & $0.584-2.451$ & & & & \\
\hline \multicolumn{3}{|c|}{ Pathological $\mathrm{T}$ factor } & & 0.230 & & & \\
\hline$-\mathrm{T} 3$ & 51 & 1.000 & & & & & \\
\hline T4- & 64 & 1.535 & $0.762-3.090$ & & & & \\
\hline \multicolumn{2}{|c|}{ Pathological $\mathrm{N}$ factor } & & & 0.554 & & & \\
\hline Negative & 24 & 1.000 & & & & & \\
\hline Positive & 91 & 1.304 & $0.541-3.141$ & & & & \\
\hline \multicolumn{3}{|c|}{ Postoperative complications } & & 0.659 & & & \\
\hline No & 87 & 1.000 & & & & & \\
\hline Yes & 28 & 1.195 & $0.543-2.631$ & & & & \\
\hline \multicolumn{3}{|c|}{ Lymph-vascular invasion } & & 0.257 & & & \\
\hline Negative & 23 & 1.000 & & & & & \\
\hline Positive & 92 & 1.732 & $0.671-4.473$ & & & & \\
\hline \multicolumn{3}{|c|}{ Pathological type } & & 0.691 & & & \\
\hline Intestinal & 68 & 1.000 & & & & & \\
\hline Diffuse & 47 & 1.146 & $0.586-2.238$ & & & & \\
\hline \multicolumn{5}{|c|}{ Lean body mass loss at 1 month after surgery 0.045} & & & 0.045 \\
\hline$<5 \%$ & 52 & 1.000 & & & 1.000 & & \\
\hline$\geq 5 \%$ & 63 & 2.076 & $1.016-4.239$ & & 2.076 & $1.016-4.239$ & \\
\hline
\end{tabular}

DG: distal gastrectomy, TG: total gastrectomy

\section{Discussion}

We previously demonstrated that a lean body mass loss of $\geq 5 \%$ at 1 month after gastrectomy was a significant risk factor for the continuation of adjuvant chemotherapy with S-1 in patients with stage II/III gastric cancer. However, whether or not the lean body mass loss influenced the survival of these patients was unclear. In this study, we found for the first time that a lean body mass loss of $\geq 5 \%$ at 1 month after gastrectomy was a significant independent risk factor for the RFS. In addition, the patients with a lean body mass loss of $\geq 5 \%$ had significantly higher rates of hematological recurrence than those with a lean body mass loss of $<5 \%$. These results indicated that the patients with a lean body mass loss $\geq 5 \%$ at 1 month after gastrectomy had a poor survival. A prospective cohort study is necessary to confirm whether or not the lean body mass loss after gastrectomy affects the gastric cancer survival.

Table 3. Comparison the sites of recurrence between the LBM loss $<5 \%$ and the LBM loss $5 \%$ groups.

\begin{tabular}{llll}
\hline & $\begin{array}{l}\text { LBM loss }<5 \%(\%) \\
(\mathrm{N}=52)\end{array}$ & $\begin{array}{l}\text { LBM loss } \geq 5 \%(\%) \\
(\mathrm{N}=63)\end{array}$ & P value \\
\hline Peritoneal recurrence & $4(7.7)$ & $6(9.5)$ & 0.729 \\
Lymph node recurrence & $3(5.8)$ & $4(6.3)$ & 0.897 \\
$\begin{array}{l}\text { Hematological } \\
\text { recurrence }\end{array}$ & $4(7.7)$ & $11(17.4)$ & 0.122 \\
\hline LBM: lean body mass & & &
\end{tabular}

LBM: lean body mass

First, we want to discuss the relationship between the lean body mass loss and the RFS. There are several possible reasons why the lean body mass loss at one month after gastrectomy affected the RFS in the locally advanced gastric cancer patients. It is most likely that the patients with a lean body mass loss $\geq 5 \%$ received little benefit from adjuvant 
chemotherapy with S-1. Similar results have been observed in our previous reports. We recently found that the body weight loss (BWL) at one month after gastrectomy was an important risk factor for the survival in patients who underwent curative D2 surgery and were pathologically diagnosed with stage II or III gastric cancer who received postoperative adjuvant chemotherapy with S-1 ${ }^{11}$. Therefore, the lean body mass loss after gastrectomy might lead to a poor survival through poor compliance with adjuvant chemotherapy with S-1. On the other hands, the loss of the lean body itself might have some clinical impacts for RFS. However, the mechanism is unclear.

However, there are some differences that should be noted between the present study and the previous study. First, the six-month continuation rate of S-1 treatment was different between these two studies. Although it was not the same cohort, the previous report found that the 6-month continuation rate of S-1 treatment was $91.7 \%$ in the patients with a loss of lean body mass $<5 \%$ and $66.3 \%$ in those with a loss of lean body mass $\geq 5 \%$. In contrast, the 6 -month continuation rate of S-1 treatment was $66.4 \%$ in the patients with a BWL $<15 \%$ and $36.4 \%$ in those with a BWL $\geq 15 \%$. Therefore, the clinical influence of these losses on the continuation rate of S-1 treatment might different. Second, the sites of first relapse were different between the two studies. On comparing the sites of first relapse in the previous study, peritoneal recurrence was more frequent in the patients with a BWL $\geq 15 \%$ than in the patients with a BWL of $<15 \%$, suggesting that S-1 was not effective in patients with severe weight loss. Of note, the ACTS-GC trial indicated that S-1 exerts a prophylactic effect to prevent peritoneal metastasis and lymph node metastasis. In contrast, in the present study, on comparing the sites of first relapse, the incidence of hematological recurrence was marginally significantly higher in the lean body mass loss $\geq 5 \%$ group than in the lean body mass loss $<5 \%$ group, while the incidences of peritoneal metastasis and lymph node metastasis were almost similar. These present study results contrasted with those from the previous study and ACTS-GC trial.

We next want to discuss the lack of a significant relationship between the lean body mass loss and the OS. Some discrepancies were observed in the findings for the RFS and OS in our study. The difference in the RFS reached significance between the lean body mass loss $\geq 5 \%$ group and the lean body mass loss $<5 \%$ group, while it did not reach significance in the OS between the 2 groups. Several reasons may explain this discrepancy. First, the mean follow-up period in our series was 40 months. Although recurrence reportedly occurs within two to three years after surgery, our follow-up period may not have been sufficient to allow definite conclusions to be drawn for the OS. This difference might have become more significant if long-term follow-up had been performed. Second, treatments after recurrence, especially chemotherapy and targeted therapy, prolong the survival of these patients. Indeed, chemotherapy and/or targeted therapy for recurrence have been reported to prolong the OS in several studies ${ }^{16-18}$. However, the details of the treatments performed after recurrence in those cases were unclear.

There are some limitations associated with this study. First, this was a retrospective single-center study. Second, there is a possibility of selection bias in this series. We cannot deny the possibility that our findings were observed by chance. Considering these limitations, our results should be validated in a different series.

In summary, the loss of lean body mass at one month after gastrectomy, which is strongly associated with poor S-1 compliance, was an important risk factor for the RFS. A multi-center prospective cohort study is necessary to confirm whether or not the lean body mass loss affects the gastric cancer survival.

\section{Acknowledgments}

This work was supported, in part, by the non-governmental organization Kanagawa Standard Anti-cancer Therapy Support System, Dr. Masumi Kamachi (Tokyo Shinagawa Hospital), Dr. Ryuji Tominaga (Fukuoka Wajiro Hospital), Dr. Nobuko Yoshiki (Yoshiki Dermatology Clinic Ginza). The authors express their sincere gratitude to Ms. Natsumi Sato and Ms. Rika Takahashi for their excellent data management in this study.

\section{Competing Interests}

The authors have declared that no competing interest exists.

\section{References}

1. Torre LA, Bray F, Siegel RL, Ferlay J, Lortet-Tieulent J, Jemal A. Global cancer statistics, 2012. CA Cancer J Clin. 2015; 65: 87-108.

2. Aoyama T, Yoshikawa T. Adjuvant therapy for locally advanced gastric cancer. Surg Today. 2017; 47: 1295-1302.

3. Yoshikawa T, Rino Y, Yukawa N, Oshima T, Tsuburaya A, Masuda M. Neoadjuvant chemotherapy for gastric cancer in Japan: a standing position by comparing with adjuvant chemotherapy. Surg Today. 2014; 44: 11-21.

4. Sakuramoto S, Sasako M, Yamaguchi T, Kinoshita T, Fujii M, Nashimoto A, et al. Adjuvant chemotherapy for gastric cancer with S-1, an oral fluoropyrimidine. N Engl J Med 2007; 357:1810-20.

5. Bang YJ, Kim YW, Yang HK, Chung HC, Park YK, Lee KH, et al. Adjuvant capecitabine and oxaliplatin for gastric cancer after D2 gastrectomy (CLASSIC): a phase 3 open-label, randomised controlled trial. Lancet 2012; 379: 315-21.

6. Cunningham D, Allum WH, Stenning SP, Thompson JN, Van de Velde CJ, Nicolson $M$, et al. Perioperative chemotherapy versus surgery alone for resectable gastroesophageal cancer. N Engl J Med 2006; 355: 11-20.

7. Schuhmacher C, Gretschel S, Lordick F, Reichardt P, Hohenberger W, Eisenberger $\mathrm{CF}$, et al. Neoadjuvant chemotherapy compared with surgery alone for locally advanced cancer of the stomach and cardia: European 
Organisation for Research and Treatment of Cancer randomized trial $40954 . J$ Clin Oncol. 2010; 28: 5210-8.

8. Macdonald JS, Smalley SR, Benedetti J, Hundahl SA, Estes NC, Stemmermann $\mathrm{GN}$, et al. Chemoradiotherapy after surgery compared with surgery alone for adenocarcinoma of the stomach or gastroesophageal junction. $N$ Engl J Med 2001; 345: 725-30.

9. Aoyama T, Kawabe T, Fujikawa $\mathrm{H}$, Hayashi T, Yamada T, Tsuchida $\mathrm{K}$ Yukawa N, Oshima T, Rino Y, Masuda M, Ogata T, Cho H, Yoshikawa T. Loss of Lean Body Mass as an Independent Risk Factor for Continuation of S-1 Adjuvant Chemotherapy for Gastric Cancer. Ann Surg Oncol. 2015; 22: 2560-6.

10. Bonadonna G, Valagussa P. Dose-response effect of adjuvant chemotherapy in breast cancer. N Engl J Med 1981; 304:101-105.

11. Aoyama T, Sato T, Maezawa Y, Kano K, Hayashi T, Yamada T, Yukawa N, Oshima T, Rino Y, Masuda M, Ogata T, Cho H, Yoshikawa T. Postoperative weight loss leads to poor survival through poor S-1 efficacy in patients with stage II/III gastric cancer. Int J Clin Oncol. 2017; 22: 476-483.

12. Japanese Gastric Cancer Association. Japanese classification of gastric carcinoma: 3rd English edition. Gastric Cancer 2011; 14: 101-12.

13. Japanese Gastric Cancer Association. Japanese gastric cancer treatment guidelines 2010 (ver. 3). Gastric Cancer 2011; 14: 113-23.

14. Yamada $T$, Hayashi $T$, Cho $H$, Yoshikawa $T$, Taniguchi H, Fukushima R, et al. Usefulness of enhanced recovery after surgery protocol as compared with conventional perioperative care in gastric surgery. Gastric Cancer 2012; 15 : 34-41.

15. Yoshikawa T, Terashima M, Mizusawa J, Nunobe S, Nishida Y, Kaji M, et al. A randomized phase III trial comparing 4 courses and 8 courses of S- 1 adjuvant chemotherapy for p-stage II gastric cancer: JCOG1104 (OPAS-1). Annals of Oncology, 2017; 28(suppl5). https://doi.org/10.1093/annonc/mdx369.010

16. Bang YJ, Van Cutsem E, Feyereislova A, Chung HC, Shen L, Sawaki A, et al. ToGA Trial Investigators. Trastuzumab in combination with chemotherapy versus chemotherapy alone for treatment of HER2-positive advanced gastric or gastro-oesophageal junction cancer (ToGA): a phase 3, open-label, randomized controlled trial. Lancet. 2010;376:687-97.

17. Wilke H, Muro K, Van Cutsem E, Oh SC, Bodoky G, Shimada Y, et al Ramucirumab plus paclitaxel versus placebo plus paclitaxel in patients with previously treated advanced gastric or gastro-oesophageal junction adenocarcinoma (RAINBOW): a double-blind, randomised phase 3 trial. Lancet Oncol. 2014; 15: 1224-35.

18. Fuchs CS, Tomasek J, Yong CJ, Dumitru F, Passalacqua R, Goswami C, et al. Ramucirumab monotherapy for previously treated advanced gastric or gastro-oesophageal junction adenocarcinoma (REGARD): an international, randomised, multicentre, placebo-controlled, phase 3 trial. Lancet. 2014; 383: 31-39. 